\title{
Tensor and Effective Vector Approach to Gravitational Radiation in the Weak Field Limit
}

\author{
P. Christillin \\ Dipartimento di Fisica, Università di Pisa, Pisa, Italy \\ Email:paolo.christillin@unipi.it
}

How to cite this paper: Christillin, P. (2021) Tensor and Effective Vector Approach to Gravitational Radiation in the Weak Field Limit. Journal of Modern Physics, 12, 798805.

https://doi.org/10.4236/jmp.2021.126051

Received: March 21, 2021

Accepted: May 10, 2021

Published: May 13, 2021

Copyright ( 2021 by author(s) and Scientific Research Publishing Inc. This work is licensed under the Creative Commons Attribution International License (CC BY 4.0).

http://creativecommons.org/licenses/by/4.0/

\section{(c) (i) Open Access}

\begin{abstract}
The purpose of the present paper is to enquire whether General Relativity (GR) is necessary for the prediction of gravitational waves. It will be shown that in the weak field limit the same predictions come also from the treatment of a zero mass, spin 2 gravitational scattering amplitude. This will also justify the simpler effective vector approach of the author, only the angular distribution differing from that of a tensor theory.
\end{abstract}

\section{Keywords}

Gravitational Waves, Weak Field Limit, Non GR Approaches

\section{Introduction}

The recent detection of gravitational waves has been indeed a great technological achievement. As regards its theoretical interpretation however some clarifying remarks are needed. As well known a considerable number of problems arose with the treatment of gravitational waves in the non linear formulation of GR which led Einstein [1] to deny their existence even in the linearized case. Those problems were finally overcome (they were even at the very beginning [2]) but the folklore survived of the "non existence of gravitational waves". This probably explains the amount of enthusiasm for their actual detection. In any case their existence in the weak field case (binary system $1916+13$ ) is out of question and justifies the validity of the linearized theory. They do indeed carry energy and the radiated power has been accounted in a simple way just from the e.m. analog [3]. Actually even in the LIGO/VIRGO experiment a linearized approach has been used to describe the detection plus a simulation for the strong emission process. 


\section{The Scattering Amplitude}

A very simple and elegant approach for the weak gravitational waves case had been put forward by Feynman [4] but seems to have remained rather unnoticed. For that reason we want to resume it and underline that the GR results for wave detection in the weak field limit can be simply obtained from the propagation of a massless spin 2 object and Lorentz invariance of the amplitude for the exchange of a graviton. This approach reproduces at the same time other features which have been so far considered to be a crucial proof of GR, namely the factor of two for gravitational light deflection. In addition it backs up the results obtained from the "naive" gravitomagnetism approach to gravitation [3]. Let therefore follow Feynman's approach [4] starting from the electromagnetic vector case.

In both cases the basic ingredient is the consideration of the scattering amplitude (respectively photon and graviton) instead of the treatment of the vertex via the proper Lagrangian.

As it will be shown this allows a considerable simplification, essentially due just to Lorentz invariance and the features of the virtual exchanged photon and graviton will be taken over to the on shell case.

\section{The vector}

The source of e.m. is the vector current $j_{\mu}$ related to the vector potential $A_{\mu}$ by

$$
A_{\mu}=-1 / k^{2} j_{\mu}
$$

or in coordinate space

$$
A_{\mu}=-\square j_{\mu}
$$

The interaction between a current and the field i.e. $j_{\mu} A^{\mu}$ yields the interaction between the currents in the scattering amplitude $f$

$$
f=j_{\mu}^{\prime} \frac{1}{k_{\mu}^{2}} j^{\mu}
$$

which for the particular choice

$$
k^{\mu}=(\omega, 0,0, k)
$$

here $k=|\vec{k}|$, reads

$$
f=\frac{1}{\omega^{2}-k^{2}}\left[j_{4}^{\prime} j_{4}-j_{1}^{\prime} j_{1}-j_{2}^{\prime} j_{2}-j_{3}^{\prime} j_{3}\right]
$$

Current conservation

$$
k^{\mu} j_{\mu}=0
$$

i.e.

$$
j^{3}=\omega / k j^{4}
$$

allows us to put the amplitude in the form

$$
f=\frac{j_{4}^{\prime} j_{4}}{k^{2}}+\frac{1}{\omega^{2}-k^{2}}\left(j_{1}^{\prime} j_{1}+j_{2}^{\prime} j_{2}\right)
$$


The first term represents the (Fourier transform of the) well known instantaneous Coulomb potential, whereas the second the two components of plane polarized propagating waves.

Such a "miraculous" separation has been obtained with the ingredients:

1) Zero mass photon;

2) Current conservation;

3) Lorentz invariance of the amplitude.

From the scattering amplitude, the form of the vertex, taken to be equal at production and at absorption, will be inferred.

\section{The tensor}

Let us then pass over to gravitation which will be treated along the same lines. We will assume the source to be given by the energy momentum tensor since gravity is determined by energy and not by mass. This will "perturb" the Minkovski metric $g_{\mu v}$ with the addition of $h_{\mu v}$ (for an extension to higher orders, which is not our concern here, see e.g. P. Menotti [5])

$$
h_{\mu v}=-1 / k^{2} T_{\mu v}
$$

or in coordinate space

$$
h_{\mu v}=-\square T_{\mu v}
$$

Current conservation is here replaced by energy momentum conservation

$$
k^{\mu} T_{\mu v}=0
$$

with the same choice

$$
k^{\mu}=(\omega, 0,0, k)
$$

In the first term of the scattering amplitude

$$
f=T_{\mu \nu}^{\prime} 1 /\left|k_{\mu}^{2}\right| T^{\mu v}-\alpha T_{\mu \mu}^{\prime} 1 /\left|k_{\mu}^{2}\right| T^{v v}
$$

which contains all possible $(i, j=4,3,2,1)$ terms, the previous energy momentum conservation condition is used to get rid of the terms with index 3 via

$$
\omega T_{4 v}=-k T_{3 v}
$$

separating again the instantaneous part from the propagating one. Thus of the initial 10 terms six remain, respectively 3 instantaneous and 3 retarded.

Focusing on the latter ones, this might seem to imply the existence of three polarization states. One of them is however eliminated by the trace condition which disposes of the spin zero contribution by adjusting the above coefficient $\alpha$ to be equal to $1 / 2){ }^{1}$

The final expression for the total amplitude then reads

$$
\begin{aligned}
f \simeq & \frac{1}{|k|^{2}}\left[T_{44}^{\prime} T_{44}\left(1-\omega^{2} / k^{2}\right)+T_{44}^{\prime}\left(T_{11}+T_{22}\right)+\left(T_{11}^{\prime}+T_{22}^{\prime}\right) T_{44}-4 T_{41}^{\prime} T_{41}\right. \\
& \left.-4 T_{42}^{\prime} T_{42}\right]+\frac{1}{\omega^{2}-k^{2}}\left[\left(T_{11}^{\prime}-T_{22}^{\prime}\right)\left(T_{11}-T_{22}\right)-4 T_{12}^{\prime} T_{12}\right]
\end{aligned}
$$

where the pole terms represent the two degrees of freedom of the propagating ${ }^{1}$ It is easy to see that this term just corresponds to the addition of the term $-1 / 2 \eta_{\mu v} T$ to the vertex of the linearized theory. Thus Feynman's approach elegantly bypasses all of the formal gauge requirements. 
waves due to the transverse quadrupole components. Exactly the GR result (see below). The ingredients used in the derivation of the amplitude are just

1) Zero mass of the graviton;

2) Energy momentum conservation;

3) No spin zero;

4) Lorentz invariance.

In this case too the vertex can be inferred only by assuming that also at the source we have a weak field production. Its structure is more involved than in the vectors case.

The first term corresponds to the usual Newtonian attraction plus a relativistic correction.

The term $T_{44}^{\prime} T_{11}$ would lead in the case of the gravitational attraction by a fixed source $\left(T_{44}^{\prime}\right)$ of a relativistic particle, in particular a photon, to an additional factor of 2 with respect to the Newtonian result $T_{44}^{\prime} T_{44}$. This is because $T_{11}=v^{2} / c^{2} T_{44}$ so that "the energy of the photon and hence its attraction is doubled." Lorentz invariance is thus enough to produce twice the Newtonian light deflection by a fixed source.

This can also be directly read from the form of the scattering amplitude. Indeed the counter term (spin zero) is manifestly zero for the photon whereas $=-1 / 2 \frac{m^{2}}{k^{2}}$ for a N.R. massive particle. Thus the amplitude for the photon is twice the latter.

All of the other terms except the 44 ones are $O\left(v^{2} / c^{2}\right)$. However only the terms $T_{41}, T_{42}$ are linear in the velocities $v_{1}, v_{2}$, thus of a vector-vector form, so only they correspond to the usual magnetic terms namely gravitomagnetic ones. In the approximate vector description of gravity they are responsible for magnetic effects which then lead via the displacement term to radiation. They enter with a factor of 4 which means that when rephrased as an effective vector interaction the current for gravitation is

$$
\boldsymbol{j}_{G}=2 m v
$$

This might appear contradictory with the current conservation relation used above for the e.m. current but has been explicitly derived from SR arguments in an effective vector formulation of gravitation (EVG) in [3], showing that only with this factor the propagation velocity is c as explicitly used also in Feynman's formulation. The previous result comes about because once more the Lorentz constraint of the scattering amplitude allows to this order, to extract an "effective" form of the vertex. Notice also, that only for the quadrupole radiation (because of the absence of dipole) can radiation be accounted for by a true quadrupole $\left(T_{11}\right)$ in the tensor formulation and by the retardation of the gravitomagnetic terms $\left(T_{41}\right)$ in the vector theory.

In conclusion the doubling of the attraction of a photon (with respect to the Newtonian case) and the doubling of the current obtained naively from the analogous electromagnetic one $(q \rightarrow m)$ are a simple consequence of the tensor 
nature of gravity and are not results peculiar to $G R$.

In short we can summarize it by saying that the (non Lorentz invariant, to this order) gravitational current (in an equivalent vector theory) of a photon can be taken to be

$$
(2 \hbar \omega, 2 m v)
$$

where the gravitomagnetic terms are now responsible for radiation along the electromagnetic lines.

\section{Quadrupole Radiation = Tidal Effects in Minkovski Space}

Given the remarkable result that a number of supposed GR predictions can be reproduced alternatively in a much simpler way, let us now enquire also in this case what remains as a peculiar GR feature. As said before in the weak field limit just a tensor formulation yields GR results, so in this section we will be concerned only with the comparison with the effective vector approach.

In the e.m. formulation the angular distribution $(d \omega)$ of the electric quadrupole radiation is proportional to

$$
\int\left|\hat{r} \times \frac{\mathrm{d}^{3} \vec{Q}_{i j}}{\mathrm{~d} t^{3}}\right| \frac{\mathrm{d} \omega}{4 \pi}=\frac{1}{5}\left|\frac{\mathrm{d}^{3} Q_{i j}}{\mathrm{~d} t^{3}}\right|^{2}
$$

[6] where

$$
\vec{Q}_{i j}=\hat{r}_{j} Q_{i j}
$$

and $Q_{i j}$ stands for the usual traceless momentum tensor. Thus the quadrupole radiation is transverse, as it should, and is it only its angle average which is expressed in terms of the true quadrupole moment.

As regards gravitation, the traceless transverse (TT) gauge expresses the power as the time derivative of the strain tensor $h_{i j}$

$$
h_{i j} \simeq \int \frac{\mathrm{d}^{3} x^{\prime} T_{i j}}{r}
$$

where $T_{i j}$ stands for the transverse spacial components of the energy momentum tensor.

No explicit expression of $T_{i j}$ is utilized but, by using conservation of the energy momentum tensor, the usual quadrupole expression is derived:

$$
\int \mathrm{d}^{3} x^{\prime} T_{i j}=\frac{1}{c^{2}} \frac{\mathrm{d}^{2}}{\mathrm{~d} t^{2}} \int \mathrm{d}^{3} x^{\prime} T_{o o} x_{i} x_{j}
$$

this incorporates all of the dynamics, retardation etc. of $T_{\mu \nu}$ in the double derivative of the source and yields for the strain tensor $h_{i j}$

$$
h_{i j}=\frac{2 G}{3 r c^{4}} \frac{\mathrm{d}^{2} Q_{i j}}{\mathrm{~d} t^{2}}
$$

Thus the emitted power is given by its time derivative and hence by the third derivative of the quadrupole momentum tensor. This can be obtained in terms of the e.m one by replacing 


$$
W_{G}=W_{e l}\left(\frac{1}{4 \pi \varepsilon_{0}} \rightarrow G, q \rightarrow 2 m\right)
$$

where the last substitution actually corresponds to the above mentioned "effective" gravitomagnetic current.

$$
W=\frac{G}{45 c^{5}}\left|\frac{\mathrm{d}^{3} Q_{i j}}{\mathrm{~d} t^{3}}\right|^{2}
$$

Thus the transverse e.m field and the transverse graviton enter the rate only as a function of the third derivative of the respective quadrupole moment.

Let us then pass over to consider differential effects so as to ascertain how much of the tensor nature of gravitation really comes into play. Let us first of all recall that

$$
g_{P} \simeq \frac{G}{c^{3} r} \frac{\mathrm{d}^{3}\left(M l^{2}\right)}{\mathrm{d} t^{3}} \simeq \frac{G M l^{2} \omega^{3}}{c^{3}} \frac{\mathrm{e}^{i \omega t}}{r} \simeq \frac{G}{c^{3} r} \frac{M l^{2}}{T^{3}}
$$

where the term $k \cdot r$ in the exponential has already been used for the retardation necessary to derive the quadrupole, and

$$
\frac{b}{c} \simeq \frac{G}{c^{4} r} \frac{M l^{2}}{T^{2}} \simeq \frac{G M}{c^{2} r}\left(\frac{l}{c T}\right)^{2}
$$

is related to the strain tensor by

$$
\frac{\boldsymbol{b}}{c} \simeq \hat{r}_{j} \cdot h_{i j}
$$

where

$$
\boldsymbol{g}=-\frac{\mathrm{d} \boldsymbol{b}}{\mathrm{d} t}
$$

b being the velocity imparted by the wave to the apparatus. It is worth noticing the difference with respect to the static radial case. All the effect is governed by the transversality of the field. Because of the finite light velocity and its propagation time between the two extremes of the apparatus (the related laser signal)

$$
t^{\prime} \simeq t+L / c
$$

time dependent tidal effects arise, thus correcting the conclusions of [7].

In effect consider now two generic points $P$ and $P^{\prime}$, the extremes of our apparatus of length $L$. They will be subjected to the gravitational acceleration of a gravitational wave which can be analyzed in sinusoidal components, so both locally at rest in the respective free fall frames. However the acceleration at the time the gravitational wave reaches $P$ and at the time elapsed for the photon to reach $P^{\prime}$ from $P$, distance covered by light in a first approximation in time $\Delta t=L / c$, are in general different simply because

$$
\mathrm{d} g_{P} / \mathrm{d} t \neq 0
$$

In other words, considering for simplicity a transverse apparatus arm (to the propagation direction and hence collinear with the polarization vector) of length 
$L$ the time dependent acceleration at one extreme is not the same the other extreme experiences at time $t^{\prime}$ even at a wave front.

Thus by expanding in terms of $\omega L / c$, one gets for the differential acceleration

$$
g_{P^{\prime}}-g_{P} \simeq\left(\frac{\mathrm{d} g_{P}}{\mathrm{~d} t}\right) \Delta t \simeq \frac{\mathrm{d} g_{P}}{\mathrm{~d} t} \frac{L}{C}
$$

In the (sub) Newtonian regime proper to interaction with matter, one can directly use $m a=F$

$$
\frac{\mathrm{d}^{2} \Delta L}{\mathrm{~d} t^{2}}=\left(\frac{\mathrm{d} g_{P}}{\mathrm{~d} t}\right) \frac{L}{C} \simeq-\left(\frac{\mathrm{d}^{2}}{\mathrm{~d} t^{2}} b\right) \frac{L}{c}
$$

so that

$$
\frac{\Delta L}{L}=-\frac{b}{c}
$$

In the general case a factor $1-\cos \theta$ if $L$ forms such an angle with respect to $r$ intervenes.

Thus the equivalence principle in the case of radiation replaces the space tidal effects of the static case by the time dependent ones for waves

$$
\Delta r / r \Rightarrow \simeq \Delta t / T \simeq L / c T
$$

i.e. the relative variation of the radiation acceleration in the time of detection over the signal period. Notice that in this semi quantitative derivation (which is in any case totally consistent with the approximations of the standard GR treatment) nothing more than the time dependence of the acceleration and the finite velocity of light has entered.

In conclusion a time dependent tidal effect for gravitational waves is reproduced also by an effective vector theory and is again not a feature of GR, although only the (hardly measurable) angular distribution and the effects of the transverse polarizations are different in the two approaches.

\section{Conclusion}

It has been recalled that just a tensor approach to gravitation can account for gravitational waves in the weak field limit with the same GR results. The same outcome can also be obtained in an effective vector theory of gravitation. Only the angular distribution and the effects of the polarization are different and might thus provide in principle a test of the tensor approach to gravitation. In conclusion in addition to the effects (light deflection, red shift, perihelion precession) which have been regarded as peculiar to GR also the main features of radiation can be obtained with alternative simpler formulations.

\section{Acknowledgements}

It is a pleasure to thank G. Morchio for continuous discussions and for enlightening criticism throughout this work. 


\section{Conflicts of Interest}

The author declares no conflicts of interest regarding the publication of this paper.

\section{References}

[1] Einstein, A. (1916) Annalen der Physik, 354, 769-822. https://doi.org/10.1002/andp.19163540702

[2] Denson, C. (2016) Hill and Pawel Nurowski. arxiv: 1608.08673v1 [gr-qc].

[3] Christillin, P. and Barattini, L. (2012) Gravitomagnetic Forces and Quadrupole Gravitational Radiation from Special Relativity. arXiv:1205.3514v3 [physics.gen-ph].

[4] Feynman, R. (1964) Lectures on Gravitation. CRC Press, Boca Raton.

[5] Menotti, P. (2017) Lectures on Gravitation. arXiv:1703.05155v1 [gr-qc].

[6] Masachusetts Institute of Technology, Department of Physics (2005) Electromagnetic Radiation and Scattering. Physics 8.07.

http://core.csu.edu.cn/NR/rdonlyres/Physics/8-07Fall-2005/9281A8EE-B595-43DBBA3E-190BE126516D/0/radiation.pdf

[7] Christillin, P. (2015) The European Physical Journal Plus, 130, 132.

https://doi.org/10.1140/epjp/i2015-15132-3 